\title{
Quasi-optical Millimetre-wave Imaging with Bio-medical Applications
}

\author{
W. Lanigan, J.A. Murphy, R. May, R.Mahon, I. McAuley, C. Markham ${ }^{1}$, K. Humphries ${ }^{1}$, T. Ward ${ }^{1}$, S. Withington ${ }^{2}$,
}

Experimental Physics Department, National University of Ireland Maynooth, Co. Kildare, Ireland,

${ }^{1}$ Electronic Engineering Department, National University of Ireland Maynooth, Co. Kildare, Ireland,

${ }^{2}$ Cavendish Laboratory, Madingley Road, Cambridge, CB3 OHE, England. e-mail: anthony.murphy@nuim.ie

\begin{abstract}
We report on millimeter-wave imaging systems being developed for both near-field analysis and quasi-optical image processing. As well as simple near field transmission we also consider Gaussian beam mode telescope based imaging, which utilizes spatial filtering techniques from Fourier optics, for the imaging of biological samples and other applications. We also report on near-field wave-front reconstruction techniques from holography and show how the techniques can also be used for imaging the phase centre of non-standard feed antennas. Finally we briefly summarise progress on the development of Fourier gratings as a method for producing multiple images of a coherent local oscillator source. Such schemes will be necessary for the development of inexpensive multiplexers for heterodyne detection schemes for sensitive array imagers and cameras.
\end{abstract}

\section{Near field imaging and spatial filtering}

We have investigated a number of issues for terahertz medical imaging, including optimization of spatial resolution and compensated efficient quasi-optical design [1]. In tandem with our experimental approach we are developing effective computational modelling tools with which to analyze system performance [2,3]. Applying expertise in the area of Gaussian mode analysis, we are developing novel optical techniques to extract detail from our targets in an efficient manner. This work constitutes part of the development of a dual modality nearinfrared/millimeter-wave system for medical imaging purposes including wound assessment [4]. We have concentrated on developing experimental systems at $0.1 \mathrm{THz}$, for proof of concept purposes, because of the ready availability of the necessary experimental components at a reasonable cost. The image obtained using a detector array or scanning system requires sophisticated image processing techniques to recover the clearest possible image of the original sample. Preliminary studies in image processing are being explored. A system to improve the structural detail obtained uses an optical filter as illustrated in figure 1 .

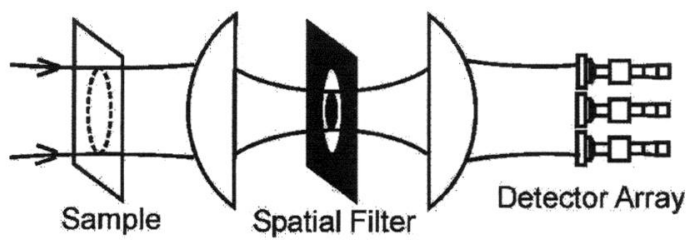

Fig. 1: Spatial filtering concept.

Using the system shown in Fig. 1. radiation scattered by edges in the target is preferentially detected, while the main on-axis illuminating beam is blocked by the central obstruction of the spatial filter. Ultimately, this will use a detector array or alternatively a scanning system (one is already developed for the Biomedical Imaging Laboratory at NUI Maynooth (GHOST)). An example of near field imaging is shown in Fig.2.

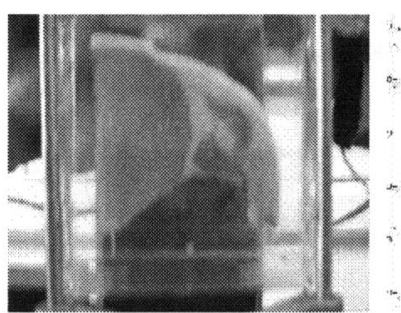

(a)

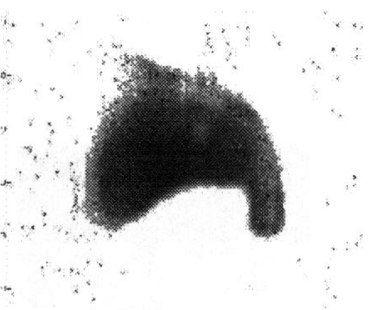

(b)
Fig. 2: Example of near field imaging of bacon slice: (a) visible picture, (b) millimetre-wave image. Dark areas represent largest areas of absorption of illuminating millimetre radiation. Some transmission through the fatty regions is observed for partially dried meat.

\section{Imaging using holographic wave-front reconstruction}

It is also possible to use wave-front reconstruction for imaging at millimeter wavelengths (MMW) employing off-axis holography $[5,6]$, a frequently used image recording technique at visible wavelengths. Holography provides a method for recording a lens-less image of an object thus reducing loss of spatial frequency information important for maximum resolution at long wavelengths. An experimental arrangement based on a simple form of near-field off-axis holography was developed, with the object illumination and reference beams derived using two radiating horn antennas fed by a single coherent source (a Gunn oscillator operating at $100 \mathrm{GHz}-3$ $\mathrm{mm}$ ) via a $3 \mathrm{~dB}$ cross-guide coupler. The reference beam was collimated using a large off-axis mirror and the hologram (or intensity pattern) resulting from the interference of the regular reference beam and the more diffuse scattered radiation from the object recorded by a scanning detector. The wave-fronts scattered by test objects were recovered by simulating reconstruction through near-field diffraction of the reference beam using the test holograms recorded over an area of $150 \mathrm{~mm}$ $\times 150 \mathrm{~mm}$ with a spatial resolution of $1 \mathrm{~mm}$.

It is possible to model the propagation of the recovered object beam including through the near-field volume between the original object and the hologram. If a plane wave illuminates the hologram located in the plane $z=0$, with an amplitude transmittance $t(x, y)$, the Fresnel-Kirchhoff integral results in the complex amplitude $\Gamma(\xi, \eta, \mathrm{d})$ at a plane at $z=d$ : 


$$
\begin{gathered}
\Gamma(\xi, \eta, d)=\frac{i a}{\lambda d} \exp \left[-\frac{\pi}{\lambda d}\left(\xi^{2}+\eta^{2}\right)\right] \\
\times \iint_{(x, y)} t(x, y) \exp \left[-i \frac{\pi}{\lambda d}\left(x^{2}+y^{2}\right)\right] \\
\quad \times \exp \left[+i \frac{2 \pi}{\lambda d}\left(x_{\xi}^{\xi}+y \eta\right)\right] d x d y,
\end{gathered}
$$

where $a$ is the amplitude of the incident plane wave. Clearly by performing an FFT of the function $t(x, y) \exp \left[-i \pi\left(x^{2}+y^{2}\right) / \lambda d\right]$ we can recover $\Gamma(\xi, \eta)$. Fig. 3 shows a reconstructed object of the shape of the letter "M." Clearly, over the small region scanned in recording the hologram, the reference beam amplitude varies only slowly and the large intensity fluctuations are dominated by the phase interference pattern produced in the presence of the illuminated object beam.

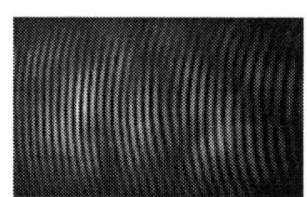

(a)

Fig. 3: Example of image reconstruction using holography: (a) measured interference hologram of illumated aperture in shape of letter " $M$ ", (b) recovered image.

The simplest demonstration of holography is where the object is a point-like source (size of order of the wavelength). As an example we can consider the interference pattern produced by the arrangement when the usual scattering object is removed and a corrugated conical pyramidal horn directly illuminated the recording plane. In this case the beam waist is small enough to be considered as a quasi-point at the phase centre being only a few wavelengths in diameter. By propagating the beam backwards from the hologram we can indentify the plane of the waist (the effective phase centre of the horn) - see Fig. 4. This is a useful inexpensive experimental method for recovering the phase centre position of a horn antenna, although the method relies on the reference beam having a true waist at the plane of the hologram. It can clearly also be applied to non-standard feeds such as lens antennas.

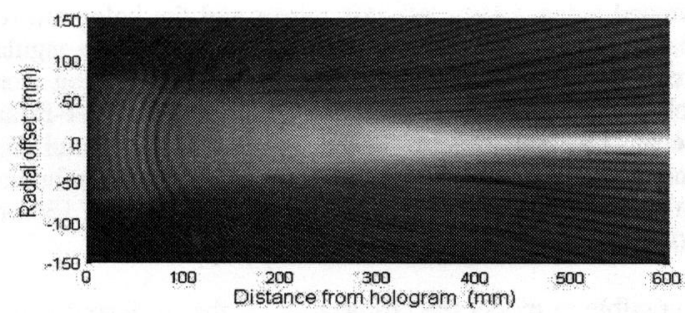

Fig. 4: Example of image reconstruction using holography: for corrugated horn antenna with aperture located at $550 \mathrm{~m}$ from the hologram plane.

\section{Array systems and phase grating multiplexers .}

Several grating types were modelled: Dammann (binary-level), discrete-level (having more than two phase levels) and Fourier (continuously-varying phase) phase gratings [7]. The gratings were designed to operate at a frequency of $100 \mathrm{GHz}(0.1 \mathrm{THz})$ the frequency range at which proof-of-concept measurements are made at NUI Maynooth. Each grating type required a different approach to find appropriate solutions for the phase profile. In the case of the Dammann grating there exists only a small number of degrees of freedom, a brute force method is used to search for solutions. Fourier gratings have a much larger number of degrees of freedom (equal to the number of sample points across the grating profile) and more sophisticated techniques are required. Phase retrieval algorithms were investigated and used to find solutions to oneand two-dimensional problems (see Fig. 5).

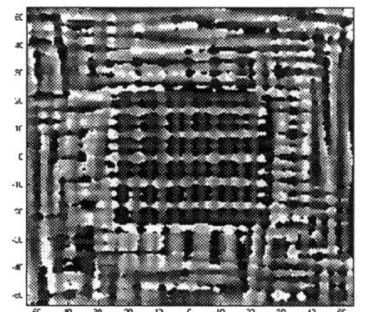

(a)

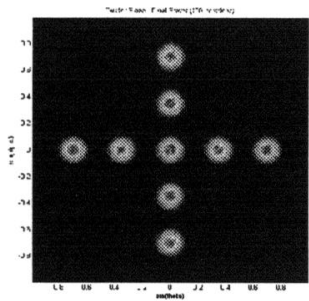

(b)
Fig. 5: Example of 2D phase retrieval: (a) Phase profile solution (left) to generate (b) a cross pattern of 9 beams.

\section{References}

[1] J. Loughran et al. 'Novel optical design for terahertz imaging applications'. Joint 29th Int. Conf. on IR and MM Waves and 12th Int. Conf. on $\mathrm{THz}$ Electronics, pp517-518, Karlsruhe, Germany, Sept 27 - Oct 1, 2004.

[2] Gradziel M.L. et al, "Modelling of millimetre-wave and Terahertz imaging systems", SPIE Optics and Photonics for Defence and Security 2004, London, UK, 25-28 October 2004, vol 5619, pp.154-165, 2004.

[3] C O'Sullivan et al, "Developments in Quasi-Optical Design for THz", Proceeding of SPIE, vol. 5498, pp. 320331,2004

[4] K. Humphries et al: "A CMOS camera-based system for clinical photoplethysmographic applications," Proceeding SPIE Conference 5823, Opto Ireland, Imaging and Vision, April 5-8, Dublin, 2005

[5] Mahon R., et al, "Terahertz holographic image reconstruction and analysis," Joint 29th Int. Conf. on IR and MM Waves and 12th Int. Conf. on THz Electronics, pp749-750, Karlsruhe, Germany.

[6] R.J. Mahon, et al., "Novel techniques for a millimeter wave imaging systems operating at $100 \mathrm{GHz}$ ", SPIE Defense and Security Symposium, Orlando Florida, USA, 28 March - 1 April 2005.

[7] May R.,et al "Phase Gratings for the sub-millimetre waveband," Joint 29th Int. Conf. on IR and MM Waves and 12th Int. Conf. on THz Electronics, pp349-350, Karlsruhe, Germany. 